\title{
LA ESTATALIDAD DEL DEPORTE INTERNACIONAL PASADO, PRESENTE, ¿FUTURO?
}

\author{
THE ROLE OF THE STATE IN INTERNATIONAL SPORT \\ PAST, PRESENT. FUTURE?
}

\author{
LA STABILITÉ DU SPORT INTERNATIONAL \\ PASSÉ, PRÉSENT ET PEUT-TRE AUSSI AVENIR
}

\author{
Carlos Pulleiro Méndez \\ Universidad del País Vasco \\ c.pulleiro.mendez@gmail.com
}

Resumen: La organización y expansión del deporte internacional durante el siglo xx se ancló principalmente a la implicación político-económica de los Estados, motivada por su representación en las competiciones deportivas por intermediación de las Federaciones Deportivas Nacionales y Comités Olímpicos Nacionales. Sin embargo, la conflictividad político-deportiva de la Guerra Fría, el cuestionamiento del papel hegemónico del Estado como centro de las Relaciones Internacionales y la propia crisis de los actos deportivos magnos ha propiciado que en la actualidad se esté socavando la centralidad del Estado en el deporte internacional.

Palabras clave: Estados, Relaciones Internacionales, actos deportivos magnos, Naciones Unidas, comercialización deportiva.

Abstract: The organization and expansion of international sport during the 20th century was anchored mainly in the political and economic involvement of nation states, motivated by their representation in sports competitions through the intermediation of the National Sports Federations and National Olympic Committees. However, the political and sports-based conflict of the Cold War, the questioning of the hegemonic role of the state as the center of International Relations, as well as the crisis faced by major sports events, has led to the current undermining of the centrality of the role of the state in international sport.

Keywords: States, International Relations, major sports events, United Nations, sports marketing.

Traducción de Gonzalo Celorio Morayta 
Résumé: L'organisation et l'épanouissement du sport international, tout au long du xxème siècle, ont dépendu surtout de l'engagement politique et économique des États, dont la participation aux jeux internationaux était assurée par les fédérations sportives et les comités nationaux olympiques. Cependant, les conflits politiques liés aux activités sportives pendant la guerre froide, le questionnement du rôle hégémonique de l'État dans les relations internationales et la crise des grandes compétitions mêmes ont contribué à affaiblir la prééminence étatique dans le sport international.

Mots clefs: États, relations internationales, grands événements sportifs, Nations Unies, commerce lié au sport.

Traducción de Bernardo Mabire

Fecha de recepción: abril de 2017 Fecha de aceptación: diciembre de 2017 
$\mathrm{E}$ N LAS RELACIONES político-diplomáticas del deporte, el olimpismo y sus juegos han sido siempre la guía de federaciones y justas deportivas. A propósito, Pierre de Coubertin, el principal impulsor del olimpismo moderno a finales del siglo XIX, sostuvo que los Juegos Olímpicos (JJ. Oo.) no podían ser solamente unos simples campeonatos mundiales; que debían ser, más bien, un medio para sofocar los conflictos mundiales, al contar con la internacionalización y democratización deportivas como sus principales herramientas para conseguirlo.

De modo tal que explícitamente el deporte se ha conceptualizado desde sus propios orígenes, más allá de su propio valor pedagógico y de desarrollo del cuerpo y mente humanos, como una herramienta política en favor de la paz entre las naciones. Pero ante esta agenda deportiva que pretende influir en la política internacional, nos encontramos también ante la agenda político-económica de los Estados, ya que, según apunta McIntosh, "si el deporte ha de influir en la política, no es fácil concebir que esa interacción vaya en una sola dirección y que la política no afecte el deporte en absoluto". ${ }^{1}$

Si bien es cierto que, según ha escrito Ramón Barba, el modelo deportivo internacional asentado en la organización del Estado explica su éxito mundial y su implantación global en detrimento de otros modelos organizativos distintos, al haber asumido un protagonismo esencial en la regulación de las práctica deportivas nacional e internacional a lo largo del siglo $\mathrm{xx},{ }^{2}$ se puede observar cómo en las últimas décadas el ámbito político y específicamente lo estatal/nacional está perdiendo centralidad en interés de otros ámbitos, actores y espacios en lo que atañe a la organización del sistema deportivo internacional, al punto de poder afirmar

${ }^{1}$ Fair Play: Ethics in Sport and Education, London, Heinemann, 1979, p. 140.

2 "Los poderes públicos y el olimpismo", Revista Jurídica del Deporte, núm. 12, 2004, p. 57. 
que el mundo del deporte de hoy en día se haya en una etapa poswestfaliana. Me refiero a aquello en lo que repara Celestino del Arenal, cuando trata de los cambios acaecidos en las Relaciones Internacionales de la Posguerra Fría, por los cuales, en la era de la globalización, la actuación estatal se ha vuelto más restringida a causa del papel de fuerzas que no se pueden controlar, constriñéndose la autonomía y la acción de no pocos gobiernos nacionales. ${ }^{3}$

En este proceso de cambio político internacional y sus implicaciones para con el deporte, cabe destacar que la capacidad transformadora de este último es menor de lo que se suele recalcar desde las propias instituciones del deporte, constituyéndose más como un reflejo de las dinámicas y agendas de los diferentes sistemas políticos internacionales vigentes que como un agente de cambio.

\section{LA CONCEPCIÓN METODOLÓGICA DE LA ESTATALIDAD}

EN EL DEPORTE INTERNAGIONAL

El sistema deportivo internacional abarca un sinnúmero de deportes y competencias que hace imposible metodológicamente examinarlo por completo en estas líneas. Sin embargo, podemos vislumbrar cómo en los albores del siglo xx el olimpismo ya se había erigido como el principal referente del mundo del deporte, convirtiéndose o directa o indirectamente en el centro de la organización deportiva internacional y ejerciendo de guía ética, diplomática y jurídica, al estar rodeado tanto de los principales actores deportivos, como políticos y económicos de carácter internacional.

No sin haber reparado en este hecho, a más de en las consideraciones de Chappelet y Klüber-Mabbott a propósito

3 "La nueva sociedad mundial y las nuevas realidades internacionales: un reto para la teoría y para la política”, en Cursos de Derecho Internacional y Relaciones Internacionales de Vitoria-Gasteiz 2001, Bilbao, Universidad del País Vasco, 2002. 
del sistema olímpico, ${ }^{4}$ se ofrece con este esquema una clara imagen de la compleja organización deportiva internacional actual:

\section{EsQUema 1}

La organización deportiva internacional actual

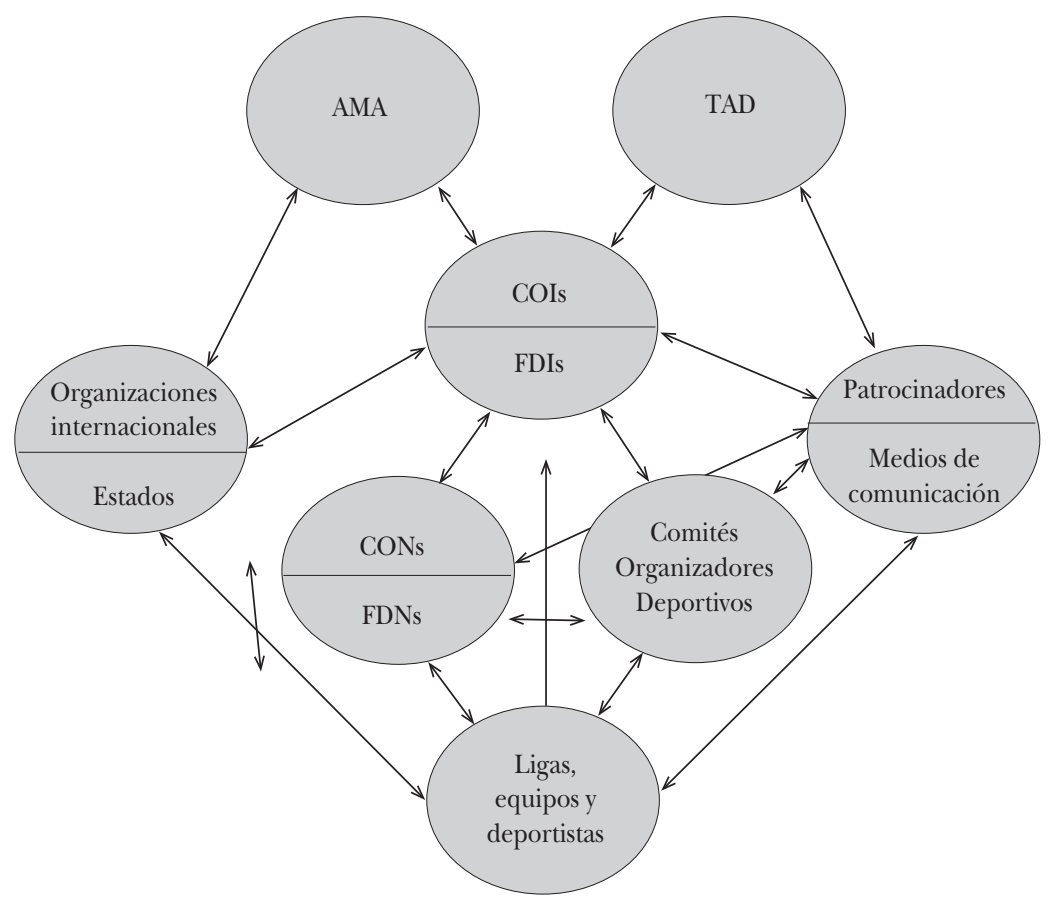

Se distingue, aquí, un núcleo compuesto por los principales estamentos deportivos, a saber: el Comité Olímpico Internacional (COI), las Federaciones Deportivas Internacionales (FDIs), los Comités Olímpicos Nacionales (CONs), las Federaciones Deportivas Nacionales (FDNs), los comités que se en-

${ }^{4}$ The International Olympic Committee and the Olympic System, Abingdon, Taylor \& Francis, 2008, p. 18. 
cargan de organizar las justas deportivas, las ligas, los equipos y los deportistas mismos.

De su parte, este núcleo se apoya en cuatro tipos de actor que han ido cobrando cada vez más relevancia, es decir los Estados y organizaciones internacionales, por un lado, y los patrocinadores y medios de comunicación, por otro, que conforman lo que podría tenerse por la periferia de la organización deportiva internacional o, por mejor decir, los medios político y económico en que se desenvuelve el núcleo. El esquema se complementa, en fin, con los dos organismos reguladores deportivos, la Agencia Mundial Antidopaje (AMA) y el Tribunal de Arbitraje Deportivo (TAD).

La realidad deportiva internacional supone un análisis complejo a la luz de las Relaciones Internacionales, ya que a pesar de que las FDIs y el COI, en cuanto organismos configuradores del ordenamiento deportivo mundial, sean "organizaciones internacionales no gubernamentales, cuya base jurídica es un acto jurídico de Derecho interno y no de Derecho Internacional Público", 5 no puede negarse que hay un claro componente de estatalidad anclado fundamentalmente en la representación del Estado ejercido por las distintas Federaciones Deportivas y Comités Olímpicos Nacionales en las justas deportivas, que constriñe los gobiernos a perseguir por todo lo ancho del mundo intereses político-económicos bajo la forma del patriotismo, la legitimidad gubernamental interna y/o externa, el prestigio internacional, la paradiplomacia, la influencia económica, entre otros, por medio de la preparación de las justas y/o la consecución de la victoria de los atletas y la obtención de medallas.

Es de considerar también que la representación de carácter estatal no ha sido estática, pues hacia 1995 el cor se arrogaba aún el derecho de reconocer los cons según sus propios

${ }^{5}$ J. J. Álvarez, J. A. Landaberea y G. Palacio, "La representación internacional del deporte federado vasco por parte de las propias federaciones deportivas vascas: viabilidad jurídica", 2014, p. 4, en http://globernance. org/wp-content/uploads/2014/04/Euskal_selekzioetako_txosten_juridikoa_gaztelania.pdf. 
términos: "un Estado, un territorio o porción de territorio que el cor considere según su absoluta decisión y como una zona de jurisdicción de un país que haya reconocido". ${ }^{6}$ Décadas antes, durante la de los 60, ya se habían precisado los conceptos nación o país, al afirmarse que ambos "se aplican por igual a una región geográfica, zona o territorio, en cuyos límites despliega su actividad o funciones un Comité Olímpico reconocido por el COI". ${ }^{7}$ La última definición de 1996, por el contrario, es mucho más explícita, pues dice de país "un Estado independiente reconocido por la Comunidad Internacional”, según se lee en el Artículo 30.1 de la Carta Olímpica. ${ }^{8}$ Se evita de este modo que el olimpismo y las FDIs, que recorrieron gran parte del camino indicado por el cor en esta materia, se conviertan en un primer interlocutor de los países que ansían una independencia política aún no reconocida internacionalmente, ${ }^{9}$ razón por la cual se optó directamente por asumir una posición conservadora y sumisa ante la realidad política internacional, poniendo en manos del Consejo de Seguridad de la Organización de las Naciones Unidas (ONU) quiénes sí y quiénes no participarán de sus justas deportivas, según se atestigua con los recientes casos de Kosovo y Sudán del Sur.

Cabe apuntar también que no todos los Estados han estado dispuestos a defender por igual sus intereses deportivos, del mismo modo en que sucede con el plano mundial, donde la igualdad estatal bajo la anarquía internacional queda en entredicho, si atendemos las capacidades y habilidades de cada entidad soberana "para ejercer un efectivo control sobre su territorio y para llevar a cabo sus objetivos". ${ }^{10}$

${ }^{6}$ M. Lora-Tamayo, La Representación internacional en el deporte, Madrid, Dykinson, 2005, p. 51.

7 O. Mayer, A través de los aros olímpicos, Madrid, coe, 1962, p. 301.

8 IOC, Olympic Charter, Lausanne, International Olympic Committee, 2015, p. 68, en https://stillmed.olympic.org/Documents/olympic_charter _en.pdf.

${ }^{9}$ Lora-Tamayo, op. cit., pp. 51-52.

10 E. Barbé, "El Estado como actor internacional: crisis y consolidación del sistema de estados", Papers: Revista de sociología, vol. 41, 
En ambos casos, lo que importa es la pericia de cada entidad para movilizar recursos y cumplir propósitos específicos, además de que su capacidad para influir en el comportamiento de otros actores del sistema se antoja esencial. En otras palabras, y como afirman Alberto Rocha y Daniel Efrén Morales, "lo relevante para el análisis no son", pues, "los intereses nacionales, sino la capacidad de los actores estatales para defender sus intereses, llegado el momento". ${ }^{11}$

\section{EL PROCESO DE LA ESTATALIZACIÓN DEPORTIVA HASTA LA SEGUNDA GUERRA MUNDIAL}

El deporte internacional no se caracteriza sino por su mutabilidad, pues se adapta a las circunstancias política y económica del momento, tanto para sobrevivir, como para expandirse por el mundo entero y ganar cada vez mayor peso en las agendas política, económica y social globales. No hay que perder de vista que el deporte moderno y su institucionalización son muy recientes, ya que sus orígenes se remontan a finales del siglo xix y en su historia descuella la creación del cor, que anima todavía la voluntad universalista de extender la práctica deportiva por todas partes: "Todos los Juegos, todas las naciones", según dijo el barón de Coubertin hacia 1911. ${ }^{12}$

Este anhelo expansionista nos indica la precariedad de la extensión y la influencia del deporte a principios del siglo xx, puesto que hasta la Primera Guerra Mundial no había sino sólo trece Federaciones Deportivas Internacionales, si se las compara con las 109 que hoy conforman la Global Association of International Sports Federations. Además, o bien por la falta de desarrollo deportivo en multitud de Estados o

1993, p. 37, en http://www.raco.cat/index.php/papers/article/viewFile/ $25163 / 58482$.

11 "Potencias medias y potencias regionales en el Sistema Político Internacional: dos modelos teóricos”, Geopolítica(s), vol. 1, núm. 2, 2010, p. 254, en http://revistas.ucm.es/index.php/GEOP/article/view/ 36329/3519.

12 Mayer, op. cit., p. 58. 
bien por motivos económicos y tecnológicos, lo cual impidió que se pagaran las costas de transportación y alojamiento en el país sede, el número de países y deportistas participantes en los escasos campeonatos internacionales era todavía muy reducido. ${ }^{13}$

Así, la mayoría de campeonatos internacionales que llegan hasta hoy día no se instituyeron sino hasta después de la Primera Guerra Mundial. Sin embargo, es de recalcar que si bien la organización de la participación era de carácter nacional/estatal, no podemos hablar todavía de una estatalidad deportiva consciente desde la parte política, ya que la cuestión deportiva era prácticamente inexistente para los poderes públicos más allá del empleo de la educación física con el propósito de la instrucción militar, como pasaba, por ejemplo, en Bulgaria, Rumania, Suecia o Alemania. A propósito, Pierre Arnaud anotó que "en todos los países europeos, al igual que en Estados Unidos, el nacimiento del deporte se debió al empeño, por una parte, de una serie de individuos aislados y, por otra, de algunos grupos privados", ${ }^{14}$ lo cual se corresponde con lo expuesto por Cesar Torres acerca de la participación, cuando afirma que ésta era "de carácter individual más que una expresión de esfuerzos colectivos nacionales". ${ }^{15}$

Pero sí que podemos encontrar cómo, desde la parte deportiva, el cor buscó siempre la estatalidad deportiva de

${ }^{13}$ Algunos de estos campeonatos fueron, por ejemplo, los de patinaje en 1896, tiro en 1897, tenis en 1900 o gimnasia en 1903. Los JJ. oo. se iniciaron en Atenas en 1896. Véase P. Arnaud, "Deporte y Relaciones Internacionales antes de 1918”, en Teresa González Aja (ed.), Sport y Autoritarismos: La utilización del deporte por el comunismo y el fascismo, Madrid, Alianza, 2002, pp. 35-37.

14 "El deporte, vehículo de las representaciones nacionales de los estados europeos", en Teresa González Aja (ed.), Sport y Autoritarismos: La utilización del deporte por el comunismo y el fascismo, Madrid, Alianza, 2002, pp. 11-12.

15 "The Latin American «Olympic explosion» of the 1920s: Causes and Consequences", The International Journal of the History of Sport, vol. 23, núm. 7, 2006, p. 1088. 
manera consciente. Hacia 1896, el miembro alemán del coI, W. Gerbhardt, sugirió la idea de crear Comités Olímpicos Nacionales "potentes", de carácter estable y definitivo, ${ }^{16}$ cuya función principal sería la de enviar a los deportistas a los Juegos Olímpicos: "de esta forma se evitarían cargas económicas y administrativas adicionales al Comité Olímpico Internacional". ${ }^{17}$

Podemos contextualizar el clima deportivo del momento y la visión política en clave estatal, si observamos también la posición de la Fédération Internationale de Football Association (FIFA) al respecto. Se rechazó, en un principio, la propuesta inglesa de 1908, según la cual las asociaciones de Irlanda y Escocia tendrían que reconocerse como federaciones independientes. Esta postura se asumió, porque de haberse permitido eso, se habría tenido que admitir también a veintiséis naciones austriacas y doce alemanas, ${ }^{18}$ a pesar de que las normas de la FIFA dictaban que se permitiera sólo una asociación por Estado. Al final, el papel dominante de la federación inglesa y el estatuto histórico de Escocia, Gales e Irlanda propiciaron su admisión entre 1910 y 1911 como asociaciones de pleno derecho. Se mantiene hasta hoy la esencia de ese acuerdo en los estatutos de la FIFA, al afirmarse en el artículo 11.6 que "con la autorización previa de la federación miembro del país del que dependa, la federación de fútbol de una región que aún no haya obtenido su independencia podrá solicitar su admisión en la FIFA". ${ }^{19}$

Sin embargo, ya en el periodo de entreguerras y según los campeonatos mundiales iban ganando relevancia por la extensión y la popularización del deporte en los diferentes

16 Mayer, op. cit., pp. 40-41.

17 Lora-Tamayo, op. cit., p. 34.

18 L. J. Harris, Britain and the Olympic Games 1908-1920: Perspectives on participation and identity, tesis, Canterbury Christ Church University, 2013, p. 109, en http://create.canterbury.ac.uk/13302/1/13302.pdf.

${ }^{19}$ FIFA, Estatutos de la FIFA, Zurich, Fédération Internationale de Football Association, 2016, p. 11, en http://resources.fifa.com/mm/document/affederation/generic/02/78/29/07/fifastatutswebes_spanish.pdf. 
países, de lo cual surgieron multitud de héroes nacionales, se fue cobrando conciencia política acerca de lo que este tipo de actos deportivos podrían ofrecer. En consecuencia, tanto el fascismo italiano como el nazismo alemán fueron pioneros, en un sentido ya explícitamente político, cuando celebraron respectivamente la Copa Mundial de fútbol de 1934 y los JJ. oo. de 1936. En ambos países "se llevaba a cabo un control recíproco del estatus [político y deportivo] que se ocupaba, lo que constituía parte de la política deportiva" ${ }^{20}$ para engrandecer aún más el patriotismo y el prestigio de ambos en todo el mundo, al haber en términos generales una implicación mucho más explícita por parte del Estado que la existente en el resto del deporte occidental.

En la Unión Soviética (URSs) la intervención estatal y la visión política del deporte también estaban implantadas desde la llegada misma de la Revolución, aunque sus motivaciones bajo el periodo estalinista de entreguerras fueron las de crear un deporte internacional comunista opuesto al deporte burgués y al fascismo, incluidos los Juegos Olímpicos.

Entrando en términos concretos de reconocimiento y participación deportiva, se profundiza en la estatalización del deporte tras la Primera Guerra Mundial por tres hechos principales: la desaparición de los denominados imperios centrales en Europa, el surgimiento de procesos secesionistas como el de Irlanda y la así llamada "explosión deportiva latinoamericana" 21 de la década de los veinte, cuando Estados como Uruguay, Argentina, Brasil o México crearon sus Comités Olímpicos Nacionales y fueron entrando poco a poco en las competiciones olímpicas y deportivas internacionales.

${ }^{20} \mathrm{R}$. Holt, "El deporte durante el periodo de entreguerras y las relaciones internacionales: algunas conclusiones", en Teresa González Aja (ed.), Sport y Autoritarismos: La utilización del deporte por el comunismo y el fascismo, Madrid, Alianza, 2002, pp. 311-324.

21 Torres, art. cit., pp. 1088-1111. 
LA CONSOLIDACIÓN DE LA ESTATALIDAD DEPORTIVA DURANTE LA GUERRA FRÍA

La llegada de la Guerra Fría comportó para el deporte, en general, y los JJ. oo., en particular, la reproducción de las lógicas del sistema político internacional del momento a causa de la rigidez de la bipolaridad en torno a la URSS y los EE.UU., al ocultar las nuevas realidades internacionales no estatales que irían surgiendo a lo largo del conflicto.

Lo relevante en la política internacional fue lo que aconteció en el sistema interestatal, ya que éste fue capaz de absorber los cambios científico-técnicos, económicos, sociales o culturales, que socavaban sus propias bases de rigidez bipolar, dotándose de una estabilidad prácticamente desconocida hasta entonces y dejando la periferia como campo abierto para el conflicto y la inestabilidad. ${ }^{22}$

En definitiva, la Guerra Fría propició las circunstancias idóneas para que el Estado se consolidara como actor predominante en las relaciones político-económicas deportivas, pero trayendo consigo conflictos e inestabilidad desconocidos hasta entonces. A fin de cuentas, el orden bipolar establecido tras la Segunda Guerra Mundial hizo de la organización de campeonatos deportivos, de las victorias, de las medallas, una muestra del poderío político y económico-social de los bloques capitalista o comunista, lo cual causó una mayor implicación de los poderes públicos en la organización deportiva, ya por su propia concepción político-deportiva en el caso de los Estados comunistas, en los que el deporte siempre fue un medio para expandir los ideales de la Revolución y mostrar al mundo los logros político-sociales que ésta había conseguido, ya por "obligación”, como sucedió en los principales Estados capitalistas de Occidente. Con respecto de los países del bloque del Este, haber dejado de ser competitivo obligó a las autoridades político-deportivas occidentales, desde finales

22 Cf. E. Barbé, Relaciones Internacionales, Madrid, Tecnos, $3^{\mathrm{a}}$ ed., 2011, pp. 310-311, y Arenal, art. cit., p. 25. 
de los años sesenta, a reaccionar con una mayor implicación financiera y de gestión deportiva pública, en aras de mejorar sus resultados deportivos para mantenerse justo detrás de las superpotencias. Se abandonaba de este modo la conceptualización inicial según la cual el deporte era una esfera privada donde la sociedad, por medio de sus organizaciones, era quien se encargaba exclusivamente de la gestión y organización deportivas.

Las consecuencias en el deporte por la fractura Este-Oeste y su capacidad de movilizar al resto de los Estados de la periferia del sistema político internacional se evidencia especialmente en los boicots masivos en contra de la participación, emprendidos por las superpotencias en los JJ. Oo. de Moscú 1980 y Los Ángeles 1984, de los que se ausentaron respectivamente 65 y 17 cons (con cifras de participación inferiores a las de Múnich 1972). Además, su estatuto y sus capacidades político-económicas permitieron a la URSs organizar un campeonato paralelo denominado Amistad 84, en que se rompieron no pocas marcas, lo cual hizo de contrapunto de Los Ángeles 1984.

En ese clima de tensión político-deportiva constante, se cuestionó la autoridad del cor como gerente de los JJ. oo. y máxima autoridad del deporte internacional, al grado de sugerirse incluso que el control de éstos pasara a manos de la UNESCO. ${ }^{23}$

Pero más allá de la bipolaridad político-deportiva con respecto del eje Este-Oeste, hay que considerar también las relaciones deportivas en clave estatal Norte-Sur, en la cual se puede destacar el reconocimiento de toda una batería de CONs y FDNs fruto de la descolonización. ${ }^{24}$ Los países afroasiáticos reconocían especialmente en el cor una función similar al reconocimiento político por parte de la onU o, al menos, un complemento para su reconocimiento institucional internacional.

23 M. Payne, Oro olimpico, London, LID editorial, 2007, p. 22.

24 Lora-Tamayo, op. cit., p. 41. 
Esta introducción masiva de Estados supuso una alteración geopolítica en el seno del deporte internacional, ya que con la entrada progresiva de gran parte de África, Asia y Oceanía, los campeonatos mundiales y los JJ. oo. conseguían ir proyectando por primera vez una verdadera imagen deportiva global e internacionalista, al haber países de todos los continentes representados de una manera masiva.

Pero la entrada de estos Estados y sus deportistas no se acompañó de una mayor representatividad en los cargos de poder. Como afirmó en una carta el entonces presidente del COI, Avery Brundage, "entrar en el movimiento [olímpico] significa hacerlo en los Juegos, antes bien que en la toma de decisiones". ${ }^{25}$ El eurocentrismo que caracteriza las FDIs y el cor implicaba ver con recelo una democratización en la gobernanza deportiva, ya que, por un lado, desconfiaban, incluso con claros matices de racismo, de las capacidades de estos nuevos países ${ }^{26}$ y, por otro, temían que los nuevos organismos deportivos reconocidos introdujeran nuevas sensibilidades políticas con lógicas y discursos provenientes de los principios de Bandung o incluso a favor de postulados comunistas.

En esto último no iban desencaminados, ya que la llegada de los países africanos sería determinante para introducir sus puntos de vista en torno al racismo en el deporte y forzar al COI y a las FDIs a posicionarse contra Sudáfrica y Rodesia, cuestión sobre la que no se había sido firme en un principio. ${ }^{27}$ La primera vez que en el cor se trató el tema del Apartheid fue el año de 1959, cuando el delegado soviético expuso, en una reunión del comité ejecutivo, el problema de adecuación a la Carta Olímpica por parte del Comité

25 I. Henry y A.-T. Manosur, "The Development of Olympic Solidarity: West and Non-West (Core and Periphery) Relations in the Olympic World", The International Journal of the History of Sport, vol. 25, núm. 3, 2008, p. 365.

26 Idem.

27 R. Espy, The politics of the Olympic Games, with an epilogue 1976-1980, London, University of California Press, 1981, pp. 69-70. 
Olímpico de Sudáfrica. Así, a pesar de que ya en los Juegos de Tokio 1964 Sudáfrica no pudo participar, la propuesta que los cons africanos hicieron al cor sobre la total exclusión de Sudáfrica del olimpismo tuvo que esperar hasta México 1968 para que pudiera convertirse en una realidad. ${ }^{28}$

La institución de los Games of the New Emerging Forces o Juegos de las Nuevas Fuerzas Emergentes (GANEFo, según su acrónimo en inglés), celebrados en Indonesia en 1963, fue la mayor afrenta a la hegemonía y al discurso olímpico en el organigrama deportivo internacional. Esta competencia surgió básicamente a raíz de la negativa del cor de patrocinar los Cuartos Juegos Asiáticos organizados en Indonesia sin participación ni de Taiwán ni de Israel, lo que movió al presidente Sukarno a anunciar la retirada de Indonesia del Movimiento Olímpico y a decretar la organización de los GANEFO, que incluían a Asia, África, Latinoamérica y los países comunistas. ${ }^{29}$

El propósito consistía en trabajar por una organización deportiva con unas bases políticas sobre las nuevas fuerzas emergentes que luchaban en contra del imperialismo estadounidense, en particular, y de todo Occidente, en general, por lo que directamente a ciertos países, como EE.UU., Reino Unido, Israel o Taiwán, ni siquiera se los invitó.

A pesar del éxito de su primera edición, los GANEFo desaparecieron a poco de haberse instituido, ya que la uRss prefirió que fueran un festival cultural que no restase valor ni a los JJ. Oo., en cuya celebración se había propuesto superar a los EE.UU., ni al golpe de Estado que apartó a Sukarno

28 Sudáfrica fue perdiendo apoyo de carácter deportivo, incluso de sus aliados políticos más acérrimos, en vísperas de México 1968, donde su exclusión contó con el apoyo de la onu, los países del bloque del Este, los deportistas afroamericanos de EE.UU. y del propio país organizador. Véase ibidem, pp. 99-103, 126.

29 C. A. Connolly, "The politics of the Games of the New Emerging Forces (GANEFo)", The International Journal of the History of Sport, vol. 29, núm. 9, 2012, p. 1312. 
del poder en 1965, ni a la Revolución Cultural China, ${ }^{30}$ que suscitó no pocas hostilidades entre ambos países, al contribuir, a su vez, a una mayor fractura o falta de coordinación entre los miembros de Bandung.

Con todo, los GANEFo fueron el aliciente para crear tanto los Juegos Panafricanos, cuyo propósito fue dividir a los países afroasiáticos, como el programa Solidaridad Olímpica, con el cual se quiso reducir las diferencias que en el desarrollo deportivo había entre los Estados del centro y la periferia.

LA POSGUERRA FRÍA Y EL REPLIEGUE DE LO ESTATAL

EN EL DEPORTE INTERNACIONAL

Según este esquema de predominio estatal en la agenda política internacional y deportiva de la Guerra Fría, sólo con la desaparición de la fractura Este-Oeste tras la caída del mundo comunista pudieron emerger una serie de fenómenos, dimensiones, estructuras, dinámicas y problemas, que habían ido aconteciendo desde mucho antes de 1991, pero los cuales no gozarían sino hasta ese entonces, con la desaparición de la bipolaridad, de una gran visibilidad y protagonismo. 31

Así, la realidad internacional se establece en la Posguerra Fría como mucho más compleja, caracterizada en la actualidad por una mayor heterogeneidad en cuanto al número de actores relevantes y con una mayor interdependencia en medio del proceso de globalización neoliberal, lo que implica notables diferencias con respecto de la naturaleza principalmente interestatal del sistema de la Guerra Fría, que se acabaría trasladando también al deporte internacional, en el que destacó el papel protagónico de los agentes económicos privados y de la ONU.

${ }^{30}$ China era el otro gran pilar de GANEFo, especialmente tras su salida del Movimiento Olímpico en 1958.

31 Arenal, art. cit., p. 25. 
Naciones Unidas como garante político de la independencia deportiva

El acercamiento a las organizaciones internacionales por parte de las autoridades deportivas a finales del siglo xx ha contribuido también a reconfigurar el papel de los Estados en el deporte internacional, uno de cuyos hechos más visibles es, sin duda, el protagonismo que han ido adquiriendo las Naciones Unidas en el sistema olímpico, en particular, y en la organización deportiva, en general, al grado de que el reconocimiento de nuevos Comités Olímpicos Nacionales o Federaciones Deportivas Nacionales por parte del COI o de las FDIs dependa de que un nuevo Estado sea reconocido por la ONU, según se expuso líneas arriba.

En ello, el punto de inflexión fue la llegada de Samaranch a la presidencia del cor en 1980, al promoverse activamente desde entonces un acercamiento con la ONU para lograr menguar por esta vía la incidencia política de los Estados en los organismos deportivos. Previamente ya hubo contactos, con el interés de Naciones Unidas por incluir al deporte en la lucha contra el Apartheid, en Sudáfrica. A su vez, el cor veía con buenos ojos este acercamiento por su deseo de reconocimiento internacional en el marco de división bipolar comunismo-capitalismo, que minimizaba la capacidad de maniobra de la máxima autoridad olímpica frente a las respectivas autoridades políticas nacionales.

Sin embargo, Naciones Unidas, durante la Guerra Fría, era todavía una organización demasiado frágil para que estos contactos no pasaran de lo puntual o influyeran no poco en lo deportivo, ya que en el Consejo de Seguridad de la ONU se mantuvieron las lógicas del sistema político westfaliano, por el cual los Estados vencedores de la Segunda Guerra Mundial plasmaron su voluntad de liderazgo, es decir las lógicas del principio de efectividad: el poder estatal de las principales potencias establece el derecho internacional. De tal suerte que el principio de efectividad siguió prevaleciendo sobre el de legalidad, que se buscaba inaugurar en la Asamblea 
General, y si bien el derecho de veto hizo las veces de válvula de seguridad, el carácter bipolar del sistema de Guerra Fría acabaría por paralizar y minar la credibilidad en el Consejo de Seguridad, a lo cual hay que añadir la falta de capacidad vinculante de la propia Asamblea General. ${ }^{32}$

Tal situación cambió con la llegada de la Posguerra Fría, una vez finalizada la bipolaridad del sistema político internacional previo. Así, se agudiza, a partir de 1992, lo que podemos denominar como transnacionalización olímpica, cuando incluyó la onU la propuesta de recuperación de la Tregua Olímpica y la resolución por la cual se estableció el Año Internacional del Deporte y el Ideal Olímpico, en 1994. La colaboración con el cor se fue extendiendo desde entonces hasta casi la totalidad de agencias de la ONU, COMO UNICEF, UNESCO, PNUD, FAO, OMS, OIT, entre otras, ya que "el olimpismo es una escuela de democracia. Existe un punto natural de unión entre la ética de los Juegos Olímpicos y los principios fundamentales de las Naciones Unidas", según el otrora presidente de la ONU, Kofi Annan. ${ }^{33}$

Se constituyen, por tanto, una gran variedad de Comisiones Olímpicas que pretenden la extensión del deporte por todo el globo, propagando los valores olímpicos entre la juventud según los valores de la onU acerca de la paz y el desarrollo del ser humano. Así, dos de los mayores logros del olimpismo en los años recientes han sido que el CoI, primero, alcanzara el estatuto de miembro observador de la Asamblea General de Naciones Unidas en 2009 y que en 2014, segundo, la propia Asamblea General emitiera una resolución en la que se apoya "la independencia y la autonomía del deporte, así como la misión del cor en la dirección del Movimiento Olímpico". ${ }^{34}$

32 Barbé, op. cit., p. 268; Rocha y Morales, Potencias medias y potencias regionales en el sistema político internacional de Guerra Fría y Posguerra Fría. Propuesta de dos modelos teóricos, Guadalajara, Universidad, 2011, pp. 15-17.

33 Payne, op. cit., p. 124.

34 IOC, "Historic milestone: United Nations recognises autonomy of sport”, Official Website of the Olympic Movement, 3 de noviembre de 2014, en http://www.olympic.org/news. 
Con este reconocimiento internacional logrado, se protege todo el sistema olímpico de los posibles ataques provenientes de los Estados, lo cual asegura, sin duda, el futuro del Cor y de los JJ. oo., quedando lejos ya la incertidumbre surgida en décadas previas sobre su viabilidad, fruto de los boicots y la falta de financiación propia.

Fuera del olimpismo, la onu también ha celebrado acuerdos con otras FDIs, clubes y deportistas, viendo en el deporte en general más allá del olimpismo un medio para profundizar en una multitud de agendas: paz mediante el deporte, igualdad de género, educación por el deporte, la lucha contra el racismo, la pobreza, la reducción de la desigualdad económica.

\section{La comercialización deportiva como elemento reconfigurador del ámbito político-estatal en el deporte internacional}

En lo que atañe a la economía, no fue sino hasta las décadas de los setenta y ochenta del siglo pasado que el deporte comenzó a contar con fuentes de financiación considerables, quedando las FDNs y los CONs muy condicionados por entonces a causa de las decisiones que tomaban las diferentes élites político-económicas de los Estados sobre temas como subvencionar el transporte y alojamiento a los deportistas en las justas deportivas o financiar la construcción de instalaciones deportivas que las propias federaciones no podían costear.

De tal suerte que las severas limitaciones en la independencia financiera del deporte a lo largo del siglo xx, especialmente el olímpico, provocaron una restricción evidente a la hora de manejar los asuntos político-deportivos, quedando las organizaciones deportivas nacionales muy expuestas tanto por el carácter exclusivo de financiación pública en los Estados comunistas, como por sus carencias en los países capitalistas, donde las naciones industriales de Occidente tenían unos recursos adecuados, pero cuyas políticas depor- 
tivas fueron impulsadas por los principios de mercado y la división de clases. ${ }^{35}$

En estas circunstancias de exposición deportiva al poder político, fue la continua y creciente tensión estatal de la Guerra Fría la que llevó definitivamente al cor y a las FDIs y FDNs a dotarse de unos recursos financieros privados suficientes., con lo cual se pretendía minimizar las consecuencias políticas y la dependencia respecto a los Estados en todo el sistema deportivo. Este hecho terminó por ser un elemento reconfigurador del propio sistema deportivo a partir de la década de los ochenta ${ }^{36}$ o, cuando menos, lo reconoció de ese modo explícitamente el expresidente del cor, Juan Antonio Samaranch, en 2005:

Estoy convencido de que la primera condición para que una organización sea respetada en todo el mundo, para que actúe de una forma verdaderamente independiente y que no se sienta ligada a compromisos externos, debe garantizarse una autonomía financiera y debe administrar recursos suficientes que le permitan gozar de una independencia ajena al poder político. ${ }^{37}$

La FIFA, por su parte, fue una pionera. El ascenso de Joao Havelange a la presidencia del organismo en 1974 comenzó un proceso de comercialización del fútbol de carácter masivo y global por medio de la búsqueda de unos patrocinadores exclusivos en los estadios, en la vestimenta de las selecciones nacionales o para las retransmisiones deportivas. Así, en la década de los noventa, la FIFA ingresaba ya más de 250 millones de dólares provenientes principalmente de los patrocinadores y de las retransmisiones de televisión, el otro gran pilar de la financiación deportiva. En el periodo comprendido en-

35 V. Girginov, "Capitalist Philosophy and Communist Practice: The Transformation of Eastern European Sport and the International Olympic Committee", Culture, Sport, Society, vol. 1, núm. 1, 1998, pp. 118-148.

${ }^{36}$ Hubo reticencias y recelos en el coi, donde aún subsistía una actitud previsora ante la perversión económica del deporte.

37 Apud Payne, op. cit., p. 11. 
tre 2011-2014, la FIFA obtuvo 5718 millones de dólares, de los cuales el $29 \%$ provenía de la mercadotecnía y el $43 \%$, de la venta de derechos de retransmisión. ${ }^{38}$

En el olimpismo, el esquema es similar, ya que, si en los Juegos de Montreal 1976 se atrajeron 628 empresas con 42 patrocinadores oficiales que aportaron en total 7 millones de dólares, en el periodo comprendido entre 1985-1988, se consiguieron, en cambio, 96 con el patrocinio de tan sólo 9 empresas. ${ }^{39}$ El cambio de paradigma fue el surgimiento del programa The Olympic Partners (TOP), cuya responsabilidad de cumplir globalmente con los acuerdos de patrocinio entre las empresas y el Movimiento Olímpico en su totalidad asumió el cor. De entonces acá, los ingresos por publicidad aumentaron hasta alcanzar los 1000 millones de dólares, sin contar los patrocinios percibidos localmente o de cada con o de cada Comité Organizador, según se evidencia en las tablas de la siguiente página.

En el aumento de los ingresos por patrocinio, la expansión de la televisión ha sido clave, pues propició el surgimiento del llamado Tv Sport System, ${ }^{40}$ en el que todos los actores implicados ganan. Así, el sistema deportivo recauda más en anunciantes, patrocinadores e ingresos por venta de derechos de retransmisión, los medios de comunicación ingresan más por publicidad y cubren los gastos por la compra de los derechos de retransmisión, mientras que las empresas anunciantes llegan a una amplia y segmentada audiencia, al rentabilizar de ese modo la inversión publicitaria.

38 Cf. T. Kistner, FIFA mafia: la historia criminal de la organización deportiva más grande del mundo, Barcelona, Editorial Corner, 2015; y FIFA, "Informe de Finanzas 2014 FIFA", 65 Congreso de la FIFA, 28 y 29 de mayo de 2015, en http://resources.fifa.com/mm/document/affederation/administration/02/56/80/39/fr2014webes_spanish.pdf.

${ }^{39}$ Hasta entonces, los derechos de comercialización estaban repartidos entre los Cons y limitados al territorio del país que controlaban. Se trataba de una estructura terriblemente compleja para aquellas empresas interesadas en el patrocinio olímpico de carácter global. Véase Payne, op. cit., pp. 26, 91.

${ }^{40}$ J. M. Puig, Olympic marketing: historical overview: university lectures on the Olympics, Bellaterra, Centre d'Estudis Olimpics (UAB), 2006, en http://olympicstudies.uab.es/lectures/web/pdf/puig.pdf. 


\section{TABla 1}

Evolución del programa тоР

\begin{tabular}{lcccc}
\hline Cuadrienio & JJ. OO. & Patrocinadores & CONs & Ingresos (millones \$) \\
\hline $1985-1988$ & Calgary/Seúl & 9 & 159 & 96 \\
$1989-1992$ & Albertville/Barcelona & 12 & 169 & 172 \\
$1993-1996$ & Lilehammer/Atlanta & 10 & 197 & 279 \\
$1997-2000$ & Nagano/Sídney & 11 & 199 & 579 \\
$2001-2004$ & Salt Lake/ Atenas & 11 & 202 & 663 \\
$2005-2008$ & Turín/Beijing & 12 & 205 & 866 \\
$2009-2012$ & Vancouver/Londres & 11 & 205 & 950 \\
$2013-2016$ & Sochi/Río de Janeiro & 12 & 206 & 1.022 \\
\hline
\end{tabular}

TABLA 2

Evolución de los ingresos por la venta de derechos de TV en los JJ. Oo. ${ }^{b}$

\begin{tabular}{|c|c|c|c|}
\hline JJ. OO. & Ingresos de TV (millones) & JJ. OO. & Ingresos de TV (millones) \\
\hline Roma 60 & $1 ' 2 \$$ & Barcelona 92 & $636 ' 1 \$$ \\
\hline Tokio 64 & $1 ' 6 \$$ & Atlanta 96 & $898 ’ 3 \$$ \\
\hline México DF 68 & $9 ' 8 \$$ & Sídney 2000 & $1.331^{\prime} 6 \$$ \\
\hline Múnich 72 & $17 ’ 8 \$$ & Atenas 2004 & $1.494 \$$ \\
\hline Montreal 76 & $34^{\prime} 9 \$$ & Beijing 2008 & $1.739 \$$ \\
\hline Moscú 80 & $88 \$$ & Londres 2012 & $2.569 \$$ \\
\hline Los Ángeles 84 & $286^{\prime} 9 \$$ & $\begin{array}{l}\text { Sochi } 2014 \\
\text { Río } 2016\end{array}$ & $4.100 \$$ \\
\hline Seúl 88 & $402 ’ 6 \$$ & & \\
\hline
\end{tabular}

a Véanse los siguientes trabajos a cargo del Ioc: Olympic Marketing Fact File: 2015 Edition, Lausanne, International Olympic Committee, 2015, en https://stillmed.olympic.org/Documents/IOC_Marketing/olympic_ marketing_fact_file_v3_2015.pdf; y Marketing Report: Rio 2016, Lausanne, International Olympic Committee, 2017, en https://stillmed.olympic. org/media/Document\% 20Library/OlympicOrg/Games /SummerGames / Games-Rio-2016-Olympic-Games/Media-Guide-for-Rio-2016/ IOC-Marketing-Report-Rio-2016.pdf.

b $I d$. 
Para el mantenimiento de esta lógica televisiva, el componente de competir entre naciones se antoja como base fundamental, puesto que la identificación del deportista como representante de su país que propugna por medallas y éxito deportivo aumenta el interés del público. Pero esta progresiva comercialización deportiva ha propiciado que, según el vaticinio de Cazorla Prieto, ${ }^{41}$ las federaciones deportivas pasaran de ser simples organismos reguladores de lo deportivo a empresas comerciales con los agentes económicos privados, estableciéndose como una fuente de financiación indispensable mediante contratos de patrocinio y de venta de derechos de televisión, lo cual agudiza aún más la división y perpetuación entre los deportes mayoritarios y más populares, que reciben una mayor financiación privada, y los minoritarios, por cuya poca visualización estarían condenados a obtener pocos ingresos por publicidad o por venta de derechos de televisión, al quedar su financiación, su influencia y su viabilidad en manos, casi exclusivamente, de los poderes públicos de los diferentes Estados.

Houlihan y Green ${ }^{42}$ han apuntado cómo las políticas deportivas nacionales en el deporte de élite, más allá de mantener el propósito de organizar actos magnos deportivos y en ellos obtener medallas y conseguir victorias, han incorporado una lógica y visión de corte empresarial con el establecimiento de objetivos de rendimiento, una planificación estratégica, criterios de financiación basados en la probabilidad de lograr un retorno de la inversión o una selección de atletas y deportes específicos para su financiación, centrándose por lo general en aquellos con más probabilidades de obtener victorias y medallas. Los autores afirman además que la adopción de este enfoque comercial/empresarial en las políticas deportivas podría matizarse con cuestiones culturales y exigencias políticas nacionales. Pero incluso en un país como Cuba se

${ }^{41}$ Deporte y Estado, Navarra, Aranzadi, $2^{\mathrm{a}}$ ed., 2013, pp. 37-38.

42 Comparative Elite Sport Development: Systems, structures and public policy, Oxford, Butterworth-Heinemann, 2008, pp. 275-277. 
están introduciendo estos criterios comerciales, por los cuales el Estado, mediante el Instituto Nacional de Deportes, Educación Física y Recreación (INDER), se ha convertido en una suerte de agencia deportiva que representa a los atletas y entrenadores ante clientes extranjeros, a la vez que promueve las capacidades de formación y aprendizaje disponibles en Cuba. ${ }^{43}$

Sin duda, con el siglo xxi se entra de lleno en lo que podría considerarse una suerte de espectacularización deportiva, según la cual los criterios comerciales y mediáticos dictaminan el sentido del deporte más allá de su propia identidad nacional o histórica. Así, ya no sólo ciertas reglas se modifican para facilitar la retransmisión del acto y así aumentar ingresos, sino que ocasionalmente se transgrede el marco nacional de la organización a fin de que las justas se hagan con nuevos mercados, como nos muestra el Giro d'Italia 2018, que debía partir de Jerusalén, o las varias ediciones celebradas en China y Qatar de la final de la Supercopa de Fútbol de Italia en la última década.

Además, esta espectacularización deportiva anclada a la industria del deporte comporta la existencia de un cambio en las relaciones de poder deportivas. Las FDIs se ven ahora presionadas más por clubes y deportistas deseosos y/o necesitados de una mayor expansión y rendimiento comercial, ${ }^{44}$ que pretenden reajustar el formato de las competiciones para obtener una mayor rentabilidad económica, amenazando incluso con abandonar las estructuras federativas, si sus deseos no se realizan. ${ }^{45}$ En el baloncesto europeo, por ejemplo, ya se

43 T. F. Carter, "¿Hasta la victoria siempre? The evolution and future of revolutionary sport", Journal of Iberian and Latin American Research, vol. 15, núm. 2, 2009, pp. 33-34.

${ }^{44}$ Hay por lo menos 27 equipos de fútbol europeo en manos de capital extranjero, principalmente chino o árabe, que buscan rentabilizar su inversión.

${ }^{45}$ La preocupación del cor por el avance de la comercialización deportiva y la ruptura de nuevas competiciones con las FDIs ha llegado al punto de hacer un llamado en defensa de la autoridad de las federaciones 
dio la ruptura Suproliga-Euroliga a principios de este siglo, no sin haber acudido por el desaguisado a la FIBA, mientras que la Union Européenne de Football Association (UEFA) ha implementado ya reformas en vísperas de la edición del año corriente de la Champions League para aplacar la intención de la Asociación Europea de Clubes de organizar un campeonato propio auspiciado por Wanda, que en marzo de 2016 se convirtió en patrocinador de la FIFA, condición que mantendrá hasta el año de 2030.

\section{LA CRISIS DE LOS ACTOS DEPORTIVOS MAGNOS} ¿HACIA UN MARCO CONTINENTAL DE LA ORGANIZACIÓN?

La combinación de la comercialización y la transnacionalización deportivas no ha significado, con todo, la desaparición total del Estado como actor interesado y protagonista en el organigrama del deporte internacional. Mientras que la organización de actos deportivos internacionales y la participación sigan siendo de carácter estatal y que este modelo de justa deportiva entre las naciones siga contribuyendo a dar réditos económicos mediante el Tv Sport System, los gobiernos, las FDIs y el cor habrán de conservar intacta su relación de intereses.

Aun así, no se puede obviar que con respecto de la Guerra Fría, estamos en la era de la globalización posnacional, caracterizada precisamente por la pérdida de autoridad del Estado, en que o las organizaciones internacionales semejantes a la Unión Europea o los acuerdos comerciales transnacionales limitan la autoridad jurídico-política de los gobiernos, mientras que las ciudades y los diferentes tipo de gobierno regional ya están afianzados como centros político-económi-

deportivas internacionales y nacionales. Véase N. Butler, "Ioc step-up effort to protect European sports model in face of pressure from rival events", 2017, en https://www.insidethegames.biz/index.php/articles/ 1058378 . 
cos de primer orden, teniendo cada vez mayor incidencia en la toma de decisiones de la agenda global.

A la luz de estas circunstancias, podemos reafirmar que hoy en día las competiciones internacionales, como los Juegos Olímpicos, la Copa Mundial de Fútbol o cualquier otro deporte tenido por "nacional" en cada país, tienen mayor relevancia para los gobiernos que en épocas pasadas, ya que se mantienen como uno de los pocos espacios donde el ámbito político-estatal conserva su predominio a razón de la representación nacional que siguen ejerciendo las FDNs y los cons. Así, la lógica de "nosotros versus otros" sobre la que se asientan las competiciones internacionales sigue funcionando tan bien como en los albores del siglo xx.

Sin embargo, en la actualidad tampoco puede obviarse los sectores más críticos de la ciudadanía, que cuestionan la gestión y el rendimiento de los actos deportivos magnos en sus países tanto en su vertiente político-económica como deportiva. Constreñidos por la crisis económica internacional, prácticamente en cualquier parte del mundo hay una cada vez mayor oposición a la celebración de este tipo de actos deportivos, pues se los tiene por una forma de derrochar el dinero público que debería destinarse a prioridades económico-sociales de otro orden. En otras palabras, se está cuestionando entre las capas populares cuál debe ser el interés nacional en el deporte, lo cual ha provocado que candidaturas olímpicas, como las de Boston, Roma, Oslo, Estocolmo, Cracovia o Hamburgo, se hayan retirado en los últimos años por una férrea oposición social, política, a más del temor a nefastas consecuencias económicas causadas por organizar los Juegos Olímpicos.

Aunque, sin duda, la mayor visualización de este rechazo y descontento sociales se ha dado en Brasil. El país sudamericano, que organizó la Copa Mundial de la FIFA en 2014 y los JJ. oo. de 2016 en Río de Janeiro, ha supuesto precisamente un punto y aparte para la viabilidad de los actos magnos del deporte mundial, ya que ha sido la definitiva confirmación de que las implicaciones económicas, sociales e incluso políticas 
de carácter perjudicial superan con creces los beneficios de éstos, ya que por primera vez en la historia, ese campeonato de la FIFA fue "el blanco de la protesta, antes que su vehículo". ${ }^{46}$ Se trata, aquí, de lo que Zimbalist había apuntado, cuando observó que la mayoría de las infraestructuras se costean o bien por medio de reducir los servicios públicos o bien por aumentar los impuestos o bien por el endeudamiento público. ${ }^{47}$ A propósito de la financiación de los actos magnos, también Victor Matheson ${ }^{48}$ ha señalado su habitual transformación en una construcción prácticamente exclusiva de instalaciones deportivas, en cuyo proceso se privilegian inversiones que a la larga no benefician, las cuales, por tanto, quedan desvinculadas del proyecto inicial, que sí tenía una visión nacional.

De otra parte, ahí están las críticas según las cuales los actos magnos no son, en esencia, sino celebraciones privadas, pese a cuyo gasto, que es principalmente público, sirven tan sólo a los intereses de las clases mejor posicionadas. Si no se olvida, por lo demás, que los procesos de expropiación se originan con las obras de acondicionamiento para acoger los actos deportivos magnos, ${ }^{49}$ la creciente oposición social cundida por el globo no puede sorprender a nadie.

La FIFA y el COI, por tanto, han reaccionado a las turbulencias políticas, económicas y sociales recientes tanto de ca-

${ }^{46}$ J. Dorsey, "Protest and Human Rights Shape Debate on Awarding of Mega Events", Huffington Post, 17 de mayo de 2014, en http://www. huffingtonpost.com.

${ }^{47}$ Circus Maximus: The Economic Gamble Behind Hosting the Olympics and the World Cup, Washington D.C., Brookings Institution Press, 2015.

48 Apud T. Waldron, "Brazil Spent \$1 Billion More On World Cup Stadiums Than Originally Planned”, ThinkProgress, 2014, en http:/ / thinkprogress.org/sports/2014/12/05/3600006.

49 Solamente dos meses después de la concesión de los Juegos a Río en 2009, se presentó un programa para desalojar favelas, el cual, para 2015, llevó a cabo 77206 desplazamientos. Véase World Cup and Olympics Popular Committee of Rio de Janeiro, Mega-Events and Human Rights Violations in Rio de Janeiro Dossier. Rio 2016 Olympics: The Exclusion Games, 2015, p. 20, en https://comitepopulario.files.wordpress.com/2016/03/ dossiecomiterio2015_eng1.pdf. 
rácter general como deportivas, planteando agendas y planes de acción a la larga con los cuales poder mejorar su propia imagen en cuanto gestores del deporte, así como asegurar la viabilidad de sus campeonatos deportivos. Estos cambios pueden traer consigo una reducción o, al menos, un cuestionamiento, de la centralidad estatal en torno a la organización de campeonatos deportivos internacionales.

La organización continental de los actos deportivos magnos como una alternativa de la insostenibilidad

de la organización estatal

Para el COI, la inquietud sobre la viabilidad de los JJ. Oo. comportó la creación en 2014 de la Agenda Olímpica 2020, la cual pretende asegurar el futuro de éstos, atrayendo más ciudades de más países. Se quiere hacer más viable económicamente tanto el proceso de candidatura como el de organización, una vez que se haya designado la ciudad sede. Para elabora tal agenda, se han consultado, a más de las autoridades e instituciones deportivas globales, ONGs, líderes políticos, instituciones culturales y empresas.

Hay que considerar también la necesidad de mejorar la utilidad política de los JJ. oo., uno de cuyos mejores ejemplos ha sido la conformación de una delegación olímpica de refugiados durante los de Río. Con ello, se sentaron las bases de lo que puede ser un precedente histórico en lo que atañe a romper con la estatalidad deportiva de la participación, ya que la esencia del Equipo Olímpico de Refugiados se fundamenta, según ha dicho el presidente del cor, Thomas Bach, ${ }^{50}$ en que estos deportistas carecían de "un equipo nacional al que pertenecer, sin bandera para marchar y sin himno nacional", por lo que fueron recibidos en los JJ. oo. con la ban-

${ }^{50}$ IOC, "Team of Refugee Olympic Athletes (ROA) created by the IOC", Official Website of the Olympic Movement, 3 de marzo de 2016, disponible en https://www.olympic.org/news/. 
dera y con el himno olímpicos. En otras palabras, el olimpismo se ha apropiado de la identidad política nacional de estos deportistas, los cuales provenían de Estados con un CON reconocido por el cor -Sudán del Sur, Siria, República Democrática del Congo y Etiopía- y por el que podrían haber competido, lo cual supone una ruptura importante con respecto del pasado reciente del olimpismo por lo que hace a la participación, ya que históricamente tanto el CoI como las FDIs han protegido el carácter estatal de la competición deportiva de los intereses de las naciones sin Estado o incluso de deportistas en particulares. ${ }^{51}$

La FIFA siempre ha sido más opaca en su gestión, pero recientemente se ha visto forzada a maniobrar principalmente por las críticas en contra del legado de la Copa Mundial de Brasil 2014:52 por las detenciones de dirigentes de la FIFA acusados de corrupción en las designaciones de la Copa Mundial, de soborno, fraude y lavado de dinero procedente de la venta de derechos de televisión y mercadotecnia; por la pérdida de patrocinadores multimillonarios, como Castrol, Continental, Emirates y Sony; por el inmovilismo, en fin, de la FIFA ante las denuncias sobre la vulneración de los derechos laborales elementales en la organización del Mundial de Qatar. ${ }^{53}$

Así, en octubre de 2016, la Fifa lanzó el plan de acción FIFA 2.0: el futuro del fútbol, que pretende reducir los ries-

${ }^{51}$ En los años cincuenta y sesenta el cor rechazó la propuesta de reconocimiento de la Unión de Deportistas Libres de Europa del Este y de la Unión Deportiva de Cuba Libre. Mediante los deportistas exiliados, ambos organismos buscaban atacar políticamente a la Unión Soviética y sus aliados, para convertirlos en símbolo del fracaso de las sociedades comunistas. Por tanto, se privilegió la estatalidad de los cons a la no discriminación por motivos de raza, religión o política de carácter individual.

52 Este tema se trata en C. Pulleiro, "Un balance de la apuesta de Brasil por los megaeventos deportivos: de la euforia por las posibilidades de desarrollo, al cuestionamiento político-económico interno y externo", $8^{\circ}$ Congreso del Consejo Europeo de Investigaciones Sociales en América Latina, 2016, en https://www.academia.edu/27557613/.

53 Para más información sobre la corrupción en la FIFA, véase Kistner, op. cit. 
gos de la sostenibilidad de la Copa Mundial de Fútbol y reestructurar a su vez la gobernanza de este deporte. La idea básica de su documento es que la FIFA y el fútbol dejen de ser un simple reflejo de la sociedad y que se conviertan en agentes transformadores, al adoptar cuatro pilares básicos de mejora: gobernanza, transparencia, responsabilidad, diversidad.

Más allá de todos los cambios propuestos en ambos documentos, ${ }^{54}$ me interesa exponer aquí cómo la FifA y el cor barajean para sus respectivos actos magnos la posibilidad de coorganizarlos entre diferentes Estados a causa de la sostenibilidad económica. Si bien a poco será todo un reto que esto se sistematice, especialmente en el caso de los JJ. oo. por su propia esencia de ubicar en un mismo lugar lo más selecto de la juventud deportista mundial, a la larga no sería algo tan descabellado, si miramos ejemplos pasados y futuros de otros torneos deportivos internacionales.

En el caso del baloncesto, por ejemplo, Ucrania renunció en junio de 2014 a celebrar el Eurobasket 2015 de la FIBA de Europa a causa de la crisis política y económica del país. Por consiguiente, el organismo decidió repartir las sedes de los grupos entre Alemania (Berlín), Croacia (Zagreb), Letonia (Riga) y Francia (Montpellier), una de cuyas ciudades, la de Lille, se designó como la de la fase final.

En el fútbol, los precedentes pueden hallarse en la Copa Mundial de 2002, celebrada en Japón y Corea del Sur, y en las ediciones de la Eurocopa de 2000, 2008 y 2012, coorganizadas respectivamente por Países Bajos y Bélgica, Austria y Suiza, Polonia y Ucrania.

Si en el caso de la FIBA se improvisó por el conflicto ucraniano, la FIFA y la UEFA, por el contrario, optaron por la organización multinacional conscientemente, argumentándose en términos político-deportivos la promoción del fútbol en

54 Se puede acceder a ellos en las siguientes direcciones: https:// www.olympic.org/olympic-agenda-2020 y http://resources.fifa.com/mm/ document/affederation/generic/02/84/35/01/fifa_2.0_vision_s_spa nish.pdf. 
nuevos países. Se aseguraban, así, la sostenibilidad de la organización y se recalcaba la contribución del fútbol a fomentar relaciones diplomáticas armoniosas entre Estados con diferencias históricas relevantes, como en el caso de Japón y Corea del Sur. ${ }^{55}$

Con todo, esta organización de eventos deportivos, dividida en ciudades de dos países, siguió tomando conceptualmente como base fundamental el marco estatal, al tener el control ambas FDNs del proceso en sus respectivos países, y defendiendo sus intereses nacionales por medio del deporte, provocándose una conflictividad no deseada desde las instituciones deportivas. ${ }^{56}$

Sin embargo, la Eurocopa 2020, aprovechando el LX aniversario de la máxima justa futbolística europea entre naciones y su ampliación desde 2016 a 24 selecciones en su fase final, ya trabaja sobre otras bases de coorganización. Las sedes serán 12 ciudades distribuidas a lo largo de todo el continente: Ámsterdam, Bakú, Bilbao, Bucarest, Budapest, Copenhague, Dublín, Glasgow, Londres, Múnich, Roma y San Petersburgo. Aquí, el papel de cada FDN se ha reducido al de proponer ciudades/sede candidatas a la UEFA, que haría las veces de órgano gestor principal del campeonato a la inversa de los casos anteriores, en los que la UEFA y la FDN correspondientes crearon una empresa conjunta responsable de todos los aspectos operativos de la fase final del torneo. Además, conceptualmente este nuevo modelo de organización se centra

${ }^{55}$ En 2002 se tuvieron que construir 17 estadios en Japón y Corea del Sur, cuyo gasto en instalaciones deportivas ascendió por lo menos a 4400 millones de dólares. Por lo demás, la unificación de las dos candidaturas, que en un principio eran independientes, se debe no poco a la lucha interna por suceder a Joao Havelange en la presidencia de la Fifa. Véase J. Horne, "The global game of football: The 2002 World Cup and regional development in Japan", Third World Quarterly, vol. 25, núm. 7, 2004, pp. 1233-1244; además de J. Horne y W. Manzenreiter (eds.), Japan, Korea and the 2002 World Cup, London, Routledge, 2002.

${ }^{56}$ Suceso que alentó a la propia FIFA a prohibir las candidaturas conjuntas entre varios países en el periodo 2004 y 2009. Véase Horne y Manzenreiter (eds.), op. cit. 
en lo local, insertando las ciudades-sede en un marco continental y relegando de este modo el ámbito estatal/nacional a un segundo plano, según se puede observar, por ejemplo, en los videos promocionales oficiales de las ciudades-sede: en el mapa de Europa dibujado no hay fronteras estatales y no aparece en ningún lado ni la simbología estatal-nacional ni la pertenencia estatal de las ciudades anfitrionas.

No sin justificarse, la UEFA se aprovecha del LX aniversario de la Eurocopa, las dificultosas circunstancias económico-sociales por las que atraviesa el mundo, la crisis de la Unión Europea tras el BREXIT y las críticas en contra tanto de los actos deportivos magnos como de las organizaciones deportivas que los gestionan. La Eurocopa del 2020 será beneficiosa en lo político y en lo deportivo "a la hora de mostrar diversidad y amistad en Europa, y mostrar también que Este, Oeste, Norte y Sur pueden unirse", según el aserto del presidente de la UEFA. ${ }^{57}$

De tal suerte que a pesar de recalcar su excepcionalidad, la modificación esencial del modelo de organización estatal/nacional de la Eurocopa 2020 debe analizarse como una prueba para la Copa Mundial FIFA o incluso para los Juegos Olímpicos mismos, en los que se podrá justipreciar la aceptación político-social, el rendimiento económico de cada sede ${ }^{58}$ o los problemas organizativos que surjan, al poder plantearse este modelo de carácter continental en los dos actos deportivos magnos más relevantes de los tiempos que están a punto de correr en el mundo.

\section{Conclusiones}

Se ha analizado, en estas líneas, el papel del Estado en lo tocante al deporte internacional, introducido por la repre-

57 "Euro 2020 will bring football together", BBC Sport, 21 de septiembre de 2016, en http://www.bbc.com/sport/football/37427568.

58 Este modelo contribuye a la reducción de costos seleccionando sedes con buenas infraestructuras en lo que toca al deporte, transporte y telecomunicaciones. 
sentación de carácter estatal en las competencias deportivas que ejercen las respectivas Federaciones Deportivas Nacionales y Comités Olímpicos Nacionales, que, desde 1996, se identifica explícitamente con la definición de país cómo Estado reconocido por la Comunidad Internacional, haciendo del resto de expresiones políticas representadas en el deporte excepciones político-geográficas.

En su evolución, la relevancia del Estado en el deporte internacional ha sido determinada, en gran parte, por el sistema político internacional vigente. Así, el ámbito estatal fue dominante durante la Guerra Fría, cuando las fracturas en clave Este-Oeste y Norte-Sur instauraron, por un lado, una feroz competición entre los Estados de los bloques políticos capitalista y comunista por las medallas y, por otro, se dio entrada a toda una batería de Estados de África, Asia y Oceanía, fruto de la descolonización. Este hecho trajo consigo niveles de conflictividad nunca antes vistos en el mundo del deporte, lo que propició que el cor y las FDIs buscaran por intermediación de la onU y de la financiación privada de las televisiones y de los patrocinadores una forma de controlar la agenda político-estatal en el deporte.

Se saca en claro, así, que la Posguerra Fría deportiva se caracterizó precisamente por haber gozado de un mayor equilibrio entre los intereses políticos de los Estados y organizaciones internacionales, los intereses deportivos de las instituciones y organizaciones del deporte y los intereses económicos de los patrocinadores y medios de comunicación, entrelazados todos ellos en el Tv Sport System.

De este modo, se introduce la rentabilidad económica en el interés nacional del deporte. Ya no se trata tan sólo de obtener un rendimiento político por lo que toca al prestigio internacional, patriotismo u orgullo nacional, sino que también se tiene que tomar en cuenta la utilidad y la rentabilidad económicas del deporte a la hora de valorar la organización de actos deportivos y la propia organización del deporte de élite por lo que hace al costo de medallas y victorias. 
Así, el cuestionamiento actual sobre el rendimiento y la utilidad de los actos deportivos magnos puede ocasionar dentro de poco el desplazamiento del Estado como marco referencial organizativo, al sustituirse con uno continental semejante al de la Eurocopa por celebrarse en un par de años.

\section{Bibliografía}

Álvarez, Juan José, Juan Antonio Landaberea y Gonzalo Palacio, "La representación internacional del deporte federado vasco por parte de las propias federaciones deportivas vascas: viabilidad jurídica”, 2014, en http://globernance.org/wp-content/ uploads/2014/04/Euskal_selekzioetako_txosten_juridikoa_ gaztelania.pdf

Arenal, Celestino del, "La nueva sociedad mundial y las nuevas realidades internacionales: un reto para la teoría y para la política”, en Cursos de Derecho Internacional y Relaciones Internacionales de Vitoria-Gasteiz 2001, Bilbao, Universidad del País Vasco, 2002, pp. 17-86.

Arnaud, Pierre, "Deporte y Relaciones Internacionales antes de 1918”, en Teresa González Aja (ed.), Sport y Autoritarismos: La utilización del deporte por el comunismo y el fascismo, Madrid, Alianza, 2002, pp. 27-48.

, "El deporte, vehículo de las representaciones nacionales de los estados europeos", en Teresa González Aja (ed.), Sport y Autoritarismos: La utilización del deporte por el comunismo y el fascismo, Madrid, Alianza, 2002, pp. 11-26.

Barba, Ramón, "Los poderes públicos y el olimpismo", Revista Jurídica del Deporte, núm. 12, 2004, pp. 85-115.

Barbé, Esther, "El Estado como actor internacional: crisis y consolidación del sistema de estados", Papers: Revista de sociología, vol. 41, 1993, pp. 33-54, en http://www.raco.cat/index.php/papers/article/viewFile/25163/58482

, Relaciones Internacionales, Madrid, Tecnos, $3^{\text {a }}$ ed., 2011.

Butler, Nick, "IOC step-up effort to protect European sports model 
in face of pressure from rival events", 2017, en https://www. insidethegames.biz/index.php/articles/1058378

Carter, Thomas F., "¿Hasta la victoria siempre? The evolution and future of revolutionary sport", Journal of Iberian and Latin American Research, vol. 15, núm. 2, 2009, pp. 25-44.

Cazorla Prieto, Luis M., Deporte y Estado, Navarra, Aranzadi, $2^{a}$ ed., 2013.

Chappelet, Jean-Loup y Brenda Kübler-Mabbott, The International Olympic Committee and the Olympic System, Abingdon, Taylor \& Francis, 2008.

Connolly, Chris. A., "The politics of the Games of the New Emerging Forces (GANEFO)", The International Journal of the History of Sport, vol. 29, núm. 9, 2012, pp. 1311-1324.

Dorsey, James, "Protest and Human Rights Shape Debate on Awarding of Mega Events", Huffington Post, 17 de mayo de 2014, en http:/ / www.huffingtonpost.com

Espy, Richard, The politics of the Olympic Games, with an epilogue 19761980, London, University of California Press, 1981.

"Euro 2020 will bring football together", BBC Sport, 21 de septiembre de 2016, en http:/ / www.bbc.com/sport/football/37427568

FIFA, Estatutos de la FIFA, Zurich, Fédération Internationale de Football Association, 2016, en http://resources.fifa.com $/ \mathrm{mm} /$ document/affederation/generic/02/78/29/07/fifastatutswebes_ spanish.pdf

—, "Informe de Finanzas 2014 FIFA", $65^{\circ}$ Congreso de la FIFA, 28 y 29 de mayo de 2015, en http://resources.fifa.com/mm/document/affederation/administration/02/56/80/39/fr2014 webes_spanish.pdf

Girginov, Vassil, "Capitalist Philosophy and Communist Practice: The Transformation of Eastern European Sport and the International Olympic Committee", Culture, Sport, Society, vol. 1, núm. 1, 1998, pp. 118-148.

Harris, Luke J., Britain and the Olympic Games 1908-1920: Perspectives on participation and identity, tesis, Canterbury Christ Church University, 2013, en http://create.canterbury.ac.uk/13302/1/ 13302.pdf 
Henry, Ian y Manosur Al-Tauqi, "The Development of Olympic Solidarity: West and Non-West (Core and Periphery) Relations in the Olympic World", The International Journal of the History of Sport, vol. 25, núm. 3, 2008, pp. 355-369.

Holt, Richard, "El deporte durante el periodo de entreguerras y las relaciones internacionales: algunas conclusiones", en Teresa González Aja (ed.), Sport y Autoritarismos: La utilización del deporte por el comunismo y el fascismo, Madrid, Alianza, 2002, pp. 311-324.

Horne, John y Wolfram Manzenreiter (eds.), Japan, Korea and the 2002 World Cup, London, Routledge, 2002.

, "The global game of football: The 2002 World Cup and regional development in Japan”, Third World Quarterly, vol. 25, núm. 7, 2004, pp. 1233-1244.

Houlihan, Barrie y Mick Green (eds.), Comparative Elite Sport Development: Systems, structures and public policy, Oxford, Butterworth-Heinemann, 2008.

IOC, "Historic milestone: United Nations recognises autonomy of sport", Official Website of the Olympic Movement, 3 de noviembre de 2014, en http:/ /www.olympic.org/news/

, "Team of Refugee Olympic Athletes (ROA) created by the IOC", Official Website of the Olympic Movement, 3 de marzo de 2016, disponible en https://www.olympic.org/news/

, Marketing Report: Rio 2016, Lausanne, International Olympic Committee, 2017, en https://stillmed.olympic.org/media/Document\%20Library/OlympicOrg/Games / Summer-Games/Games-Rio-2016-Olympic-Games/MediaGuide-for-Rio-2016/IOC-Marketing-Report-Rio-2016.pdf , Olympic Charter, Lausanne, International Olympic Committee, 2015, en https://stillmed.olympic.org/Documents/olym pic_charter_en.pdf

, Olympic Marketing Fact File: 2015 Edition, Lausanne, International Olympic Committee, 2015, en https://stillmed.olympic. org/Documents/ IOC_Marketing/olympic_marketing_fact_fi le_v3_2015.pdf

Kistner, Thomas, FIFA mafia: la historia criminal de la organización deportiva más grande del mundo, Barcelona, Editorial Corner, 2015. 
Lora-Tamayo, Marta, La Representación internacional en el deporte, Madrid, Dykinson, 2005.

Mayer, Otto, A través de los aros olímpicos, Madrid, COE, 1962.

McIntosh, Peter, Fair Play: Ethics in Sport and Education, London, Heinemann, 1979.

Payne, Michael, Oro olimpico, London, LID editorial, 2007.

Puig, Josep M., Olympic marketing: historical overview: university lectures on the Olympics, Bellaterra, Centre d'Estudis Olimpics (UAB), 2006, en http:/ /olympicstudies.uab.es/lectures/web/ pdf/puig.pdf

Pulleiro, Carlos, "Un balance de la apuesta de Brasil por los megaeventos deportivos: de la euforia por las posibilidades de desarrollo, al cuestionamiento político-económico interno y externo", $8^{\circ}$ Congreso del Consejo Europeo de Investigaciones Sociales en América Latina, 2016, en https:/ /www.academia.edu/275 57613/

Rocha, Alberto y Daniel E. Morales, "Potencias medias y potencias regionales en el Sistema Político Internacional: dos modelos teóricos”, Geopolítica(s), vol. 1, núm. 2, 2010, pp. 251-279, en http:/ / revistas.ucm.es/index.php/GEOP/article/view/363 29/35196

$-\mathrm{y}-$ Potencias medias y potencias regionales en el sistema político internacional de Guerra Fría y Posguerra Fría. Propuesta de dos modelos teóricos, Guadalajara, Universidad, 2011.

Torres, Cesar R., "The Latin American «Olympic explosion» of the 1920s: Causes and Consequences", The International Journal of the History of Sport, vol. 23, núm. 7, 2006, pp. 1088-1111.

Waldron, Travis, "Brazil Spent \$1 Billion More On World Cup Stadiums Than Originally Planned", ThinkProgress, 2014, en http: / / thinkprogress.org/sports/2014/12/05/3600006/

World Cup and Olympics Popular Committee of Rio de Janeiro, $M e-$ ga-Events and Human Rights Violations in Rio de Janeiro Dossier. Rio 2016 Olympics: The Exclusion Games, 2015, en https://comitepopulario.files.wordpress.com/2016/03/dossiecomiterio2015_ eng1.pdf

Zimbalist, Andrew, Circus Maximus: The Economic Gamble Behind Hosting the Olympics and the World Cup, Washington D.C., Brookings Institution Press, 2015. 
